brain showed bilateral hypodensity in the putamini and globus pallidi, consistent with hemorrhagic necrosis. On Day 60, brain MRI revealed bilateral hyperintensity in the same areas, which subsequently decreased six months and two years later.

Conclusion: Cyanide poisoning may predominate over carbon monoxide poisoning in smoke inhalation. Cyanide was the most likely cause of the patient's early neurological manifestations (including apnea and hydroxycobalamin-corrected shock), and late neurological manifestations. This case also questions the dose of hydroxycobalamin that should be administered in such cases.

Keywords: cyanide; cyanide poisoning; smoke inhalation Prebosp Disast Med 2005;20(5):s157-s158.

Building Hospital-Ready Medical Surge Capacity for Disasters

Marilyn Biviano; ${ }^{1}$ Mary Duley, ${ }^{2}$ Atila Omer,; James G. Hodge

1. Health Resources and Services Administration, Department of Health and Human Services, Washington, DC USA

2. Office of Public Health Preparedness, Connecticut Department of Public Health, Hartford, Connecticut USA

3. Collaborative Fusion, Inc., Bethesda, Maryland USA

4. Center for Law and the Public's Health, Johns Hopkins Bloomberg School of Public Health, Baltimore, Maryland USA

Introduction: Building health personnel emergency surge capacity is an essential component of hospital preparedness. The Emergency System for Advance Registration for Volunteer Health Professionals (ESARVHP) Technical and Policy Guidelines, Standards, and Definitions were developed with the assistance of ten National Working Groups that provided expertise and perspective on how to address key topics confronting the National Bioterrorism Hospital Preparedness Community. Twenty-four States and several professional associations and volunteer organizations supported and contributed to the Guidelines development.

Objective: This panel presentation will describe the following: (1) the challenges in developing a national system of state-based medical personnel registries; (2) the ESAR-VHP Guidelines, Standards, and Definitions development and implementation issues; (3) emergency credential standards that have been developed for 20 hospital-based professions; (4) the legal and policy issues facing volunteers, and how states and hospitals are addressing these issues; and (5) Connecticut's hospitalbased ESAR-VHP, operated by the Connecticut Department of Public Health in conjunction with Yale New Haven Health System, and why it is a model for other states and the United States.

Keywords: Connecticut; development; disaster; emergency; health personnel; hospital; surge capacity, United States

Prebosp Disast Med 2005;20(5):s158
Active Learning, Bioterrorism Clinics, and Continuing Professional Education: An Ideal Combination Jeffrey Bratberg; ${ }^{1}$ Brett Feret, ${ }^{1}$ Stephen Kogut, ${ }^{1}$ Gregory Low, ${ }^{1}$ Alysia Mibalakos, ${ }^{2}$ Michael Simeone ${ }^{2}$

1. University of Rhode Island College of Pharmacy, Kingston, Rhode Island USA

2. Rhode Island Department of Health, Providence, Rhode Island USA

Objective: This presentation will describe the integration of active learning and emergency preparedness techniques into an annual, continuing, professional education program to enhance learning of advanced concepts in bioterrorism dispensing clinic design, operation, and evaluation.

Methods: After didactic lectures on bioterrorism agents, government roles in bioterrorism response, and pharmacist surveillance, a mock bioterrorism clinic "play" was performed on-stage using audience volunteers as actors in predetermined clinic volunteer and patient roles. To create a more realistic emergency clinic, a randomly selected portion of the audience was assigned clinical volunteer roles, and asked to set up a dispensing clinic quickly. The remaining audience members were patients, some of which were assigned roles to challenge the clinic volunteers. Program facilitators, all experts in bioterrorism clinic design and operation, provided assistance and evaluation. An open critical discussion ("hot wash") was conducted immediately following the program.

Results: During the "hot wash", some participants reported that the play, with its attendant chaos of establishing the dispensing clinic under time constraints, represented a paradigm shift from typical continuing education programs. Many participants reported that the play increased their understanding of the concepts of emerging leadership and the flexibility required in the fog of a bioterrorism emergency.

Conclusion: Continuing, professional education programs that integrate active learning techniques may facilitate practical learning of the development and operation of bioterrorism dispensing clinics. Whole audience participation may enable more voices to contribute to continuing education programs.

Keywords: bioterrorism; clinics; continuing education; leadership; learning, active; simulation

Prehosp Disast Med 2005;20(5):s158.

Development and Application of a Bioterrorism Emergency Management Plan

Jeffrey Bratberg; ${ }^{1}$ Kimberly Deady ${ }^{2}$

1. University of Rhode Island College of Pharmacy, Kingston, Rhode Island USA

2. University of Kentucky College of Pharmacy, Lexington, Kentucky USA

Objective: This presentation describes the development and application of a bioterrorism emergency management plan (EMP) during a university pharmacy course. Methods: Descriptive information was obtained from personal observations and anonymous teaching evaluations by students. 
Results: Thirty university students were assigned to participate in the development and application of a bioterrorism EMP over the course of one semester during an elective pharmacotherapy course entitled "Advanced Topics in Infectious Disease and Pulmonary Pharmacotherapy". The bioterrorism EMP project encompassed outcomes of patient and population-based care, systems management, disease prevention, promotion of public health, and the development of public health policy. Innovative elements of the bioterrorism EMP project included: (1) semester-long written, oral, and practical participation and assessment; (2) comprehensive and topical educational content; and (3) systematic, peer-reviewed, team-building efforts. The students also gained valuable experience as pharmacy-based volunteers in the largest universitybased mock dispensing clinic exercise performed to date. The class developed a nearly 100-page referenced practical EMP that was applied successfully and assessed during a formal tabletop botulism outbreak exercise. While some students expressed frustration working in large, unstructured, leader-dominated groups, teaching evaluations (and informal comments) indicated a high rate of satisfaction with this educational method.

Conclusion: Motivated university students can develop and apply a bioterrorism EMP as a group project. Meeting of project objectives may be facilitated by awareness and reinforcement of class and group expectations, smaller and more structured groups, and frequent progress evaluations by instructors and peers.

Keywords: bioterrorism; dispensing clinic; education; emergency management plan; public health; tabletop exercise Prebosp Disast Med 2005;20(5):s158-s159.

\section{Hospital Emergency Response to a Community Chemical Spill \\ Randy Casteel}

Hamilton Medical Center, Hamilton Health Care System, Dalton, Georgia USA

Introduction: Despite continuous efforts to plan for events involving weapons of mass destruction and ongoing testing of hospital incident command systems (ICS), hospital emergency preparedness for chemical emergencies still may be lacking.

Objective: This presentation describes the hospital emergency response to a community chemical spill at Hamilton Medical Center in Dalton, Georgia on 12 April 2004.

Methods: Descriptive information was obtained from observations and hospital records of the event.

Results: At 21:15 hours (h) on 12 April 2004, local emergency medical services (EMS) notified the hospital that five patients were en route to the hospital in a police car. The hospital ICS was activated, and hospital personnel began preparing for the arriving victims. Because the hospital's decontamination equipment and supplies could not be located, a makeshift decontamination area was improvised with four blankets hung between intravenous poles and a water hose attached to an outside spout. Only hospital personnel dressed in splash gowns and respiratory masks performed decontamination.

Communication by hospital radio was unreliable, due to the constant chatter and dead zones not discovered during previous testing. The hospital ICS communicated using cell phones and runners. Communication with on-scene ICS never was established and no one could be located who could identify the chemical agent responsible. At $02: 30 \mathrm{~h}$, a call came in identifying the chemical as allyl alcohol and recommending that the best decontamination method was to rinse patients with copious water. The hospital ultimately decontaminated 154 patients, including 14 police officers and six EMS workers who continued working in the hot zone without protective clothing or respirators. No deaths occurred.

Conclusion: Numerous lessons were learned from the hospital emergency response to this event, which will be applied to future hospital emergency preparedness.

Keywords: allyl alcohol; chemical spill; decontamination; emergency medical services; hospital; incident command system Prehosp Disast Med 2005;20(5): 159 .

\section{Estimating Surge Capacity for Mass-Casualty Incidents \\ Debi Drake; Chris Dent}

\section{Saint Alphonsus Regional Medical Center, Boise, Idaho USA}

Introduction: As emergency departments (EDs) address the need to generate surge capacity for a sudden influx of patients in a mass-casualty incident (MCI), guidelines are needed to determine minimum staffing levels and the types and numbers of equipment and supplies needed.

Objective: This presentation describes the development of minimum guidelines for EDs to manage a large influx of patients effectively in the first several hours after a MCI.

Methods: Descriptive information was obtained from observations and records associated with this project.

Results: The following sources of information were analyzed in the development of the minimum guidelines: (1) American College of Surgeons recommendations regarding the ED care of major trauma (blunt and penetrating injuries, burns); (2) United States' Center for Disease Control information on the most common injuries in mass trauma; (3) standard triage categories of red, yellow, and green; and (4) a survey of 250 rural and urban hospitals that found that an influx of twice the ED bed volume would be considered an MCI at each facility. A spreadsheet was developed to calculate immediate minimum staffing, equipment, and supply needs for an influx of twice the number of ED beds. This surge capacity estimation tool addresses only the needs of the MCI and not the needs of normal patient volume. In addition, this tool does not address decontamination or isolation needs.

Conclusion: This surge capacity estimation tool is a useful resource when preparing for an MCI.

Keywords: emergency department; estimation tool; hospital; masscasualty incident; needs; surge capacity

Prebosp Disast Med 2005;20(5):s159. 